\title{
High speed visualization of converging spherical shock waves in an aspherical test section
}

\author{
S. H. R. HOSSEINI and K. TAKAYAMA \\ Interdisciplinary Shock Wave Research Laboratory, Institute of Fluid Science, \\ Tohoku University, Sendai 980-8577, Japan
}

\begin{abstract}
In order to quantitatively observe spherical shock waves and the flow field behind them, an aspheric spherical transparent test section was designed and constructed. Spherical diverging shock waves were produced at the center of the spherical test section by irradiation of a pulsed Nd:YAG laser beam on micro explosives. Pressure histories at different points over the test section were measured to validate production of uniform shock waves. After reflection of spherical shock wave from the test section, a converging spherical shock wave was produced. Double exposure holographic interferometry and time resolved high speed photography were used for flow visualization. The whole sequence of diverging and converging spherical shock waves propagation and their interaction with product gases were studied.
\end{abstract}

Keywords: High speed visualization, Double exposure holographic interferometry, Converging spherical shock waves

\section{Introduction}

High temperatures and pressures can be produced at the center of convergence of spherical or cylindrical shock waves, which suggest various interesting applications. Dynamics and stability of converging cylindrical shock waves have been intensively studied experimentally and analytically. Nevertheless, production of uniform converging spherical shock waves in gases and its stability in the vicinity of the center of convergence are still open questions. In the Institute for Aerospace Studies, University of Toronto, a hemispherical implosion chamber was constructed, Glass and Sislian [1], for producing explosively driven implosion waves which were focused and provided a small region of extremely high pressures and temperatures. In the present study, an attempt has been made to produce spherical implosion waves and to study stability and convergence of spherical shock waves.

\section{Experimental method}

In order to visualize spherical shock waves and the flow field behind them, an aspheric spherical transparent test section was designed and constructed The test section has $270 \mathrm{~mm}$ by $203.3 \mathrm{~mm}$ aspherical shape outer wall and $150 \mathrm{~mm}$ diameter spherical shape inner wall and was made from transparent plastic. This lens shaped window permits the collimated visualization laser beam to traverse the test section parallel and emerge parallel. A schematic diagram of the visualization light rays in the test section is shown in Fig. 1. Shock waves were generated by explosion of $10.0 \pm 1 \% \mathrm{mg}$ silver azide pellets (AgN3; Chugoku Kayaku Co., Ltd., Japan). The charge was glued to an optical fiber and was ignited by irradiation of a pulsed Nd:YAG laser beam (1064 nm wavelength, $7 \mathrm{~ns}$ pulse duration, and $25 \mathrm{~mJ} /$ Pulse energy). Since the inner wall of the test section has spherical shape, the reflection of the diverging shock waves from the test section produced converging spherical shock waves. Double exposure holographic interferometry and time resolved high speed photography were used for flow visualization.

\section{Results and Discussion}

Figure 2 shows pressure histories, normalized by initial pressure, measured at the test section for detonation of $10.0 \mathrm{mg}$ silver azide pellet in air at initial pressure $100.65 \mathrm{kPa}$. As can be seen in experimental set up of Fig. 1, pressure transducers of $\mathrm{Ch} 1$ and $\mathrm{Ch} 2$ record reflected or face-on pressure. A photodiode was used to record a signal from Nd:YAG laser irradiation. In Fig. 2, $128 \mu \mathrm{s}$ after micro charge ignition the diverging shock wave arrives at the test section to induce a steep pressure jump from atmospheric pressure. The reflected shock overpressure $\left(\mathrm{P}_{\mathrm{r}}=\mathrm{p}_{\mathrm{r}} / \mathrm{p}_{0}\right)$ has a value of 1.8. From Rankine-Hugoniot relations the shock overpressure or side-on overpressure $\left(P_{s}=p_{s} / p_{0}\right)$ of 1.35 was computed. This corresponds to incident diverging shock wave Mach number $M s=1.14$ to the test section's wall.

Figure 3 shows infinite fringe interferograms for explosion of $10.0 \mathrm{mg}$ silver azide in air at atmospheric pressure of $100.2 \mathrm{kPa}, 100 \mu$ s after the explosion. The diverging shock wave is propagating toward the test section's wall and fringes or iso-density spherical counters behind the primary shock wave were observed. A jet of product gases in the ignition direction was produced, which disturbed the spherical flow filed, and the leading or disturbed part of shock wave in that direction has reflected from the test section and would decisively affect implosion of primary shock wave. The secondary shock wave is also well observed in this hologram.

In order to produce uniform spherical diverging shock waves, a two directional ignition system was employed. By splitting the igniting $\mathrm{Nd}$ :YAG laser 
beam, two optical fibers were used. Figure 4 shows one frame of high speed shadowgraph of converging spherical shock wave. Propagation of imploding shock wave through high temperature product gases caused an elliptical shape of shock front. A comparison between Ray shock theory, Whitham [2], and experimental shock speed was made and reasonable agreement was obtained.

\section{Conclusions}

In order to produce spherical converging shock waves an aspherical shaped transparent test section with a spherical cavity was designed and constructed. By explosion of micro charges, spherical diverging shock waves were generated. Pressure histories at two facing points over the test section were measured. Double exposure holographic interferometric quantitative flow visualization and time resolved high speed shadowgraph methods were performed. In order to produce uniform shock waves, micro charges were ignited in two directions and convergence of spherical shock waves were studied.

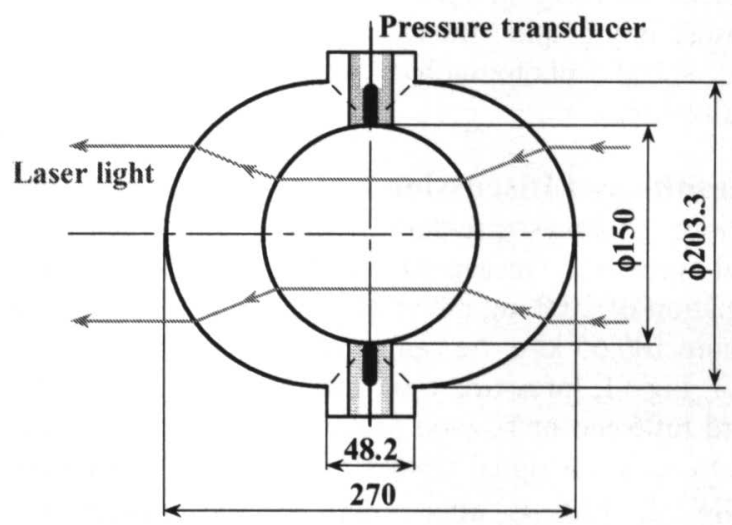

Fig. 1 Schematic diagram of the laser light rays in the aspheric spherical transparent test section

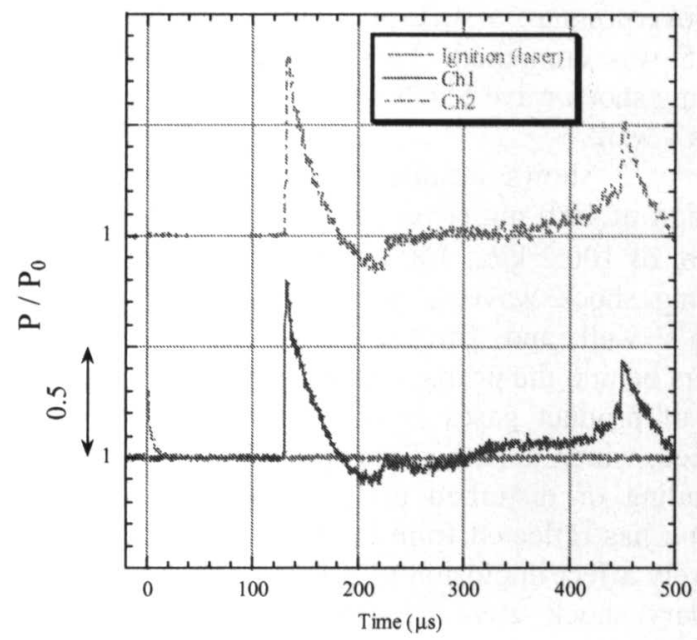

Fig. 2 Pressure histories measured at the test section and normalized by the initial pressure $\mathrm{P}_{0}=100.65 \mathrm{kPa}$

\section{References}

1) Glass, I.I., Sislian, J.P.: Nonstationary Flows and Shock Waves, Oxford Science Publications, (1994).

2) Whitham, G.B.: Linear and Nonlinear Waves, WileyInterscience, (1973).

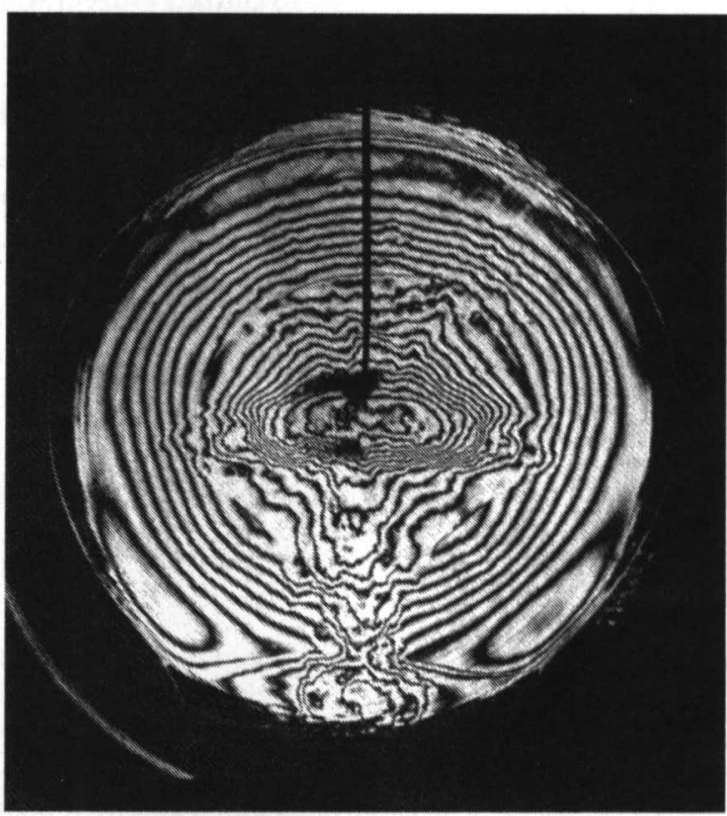

Fig. 3 Infinite fringe interferogram of diverging spherical shock wave $100 \mu$ s after explosion of 10.0 $\mathrm{mg}$ silver azide charge at ambient pressure $100.2 \mathrm{kPa}$

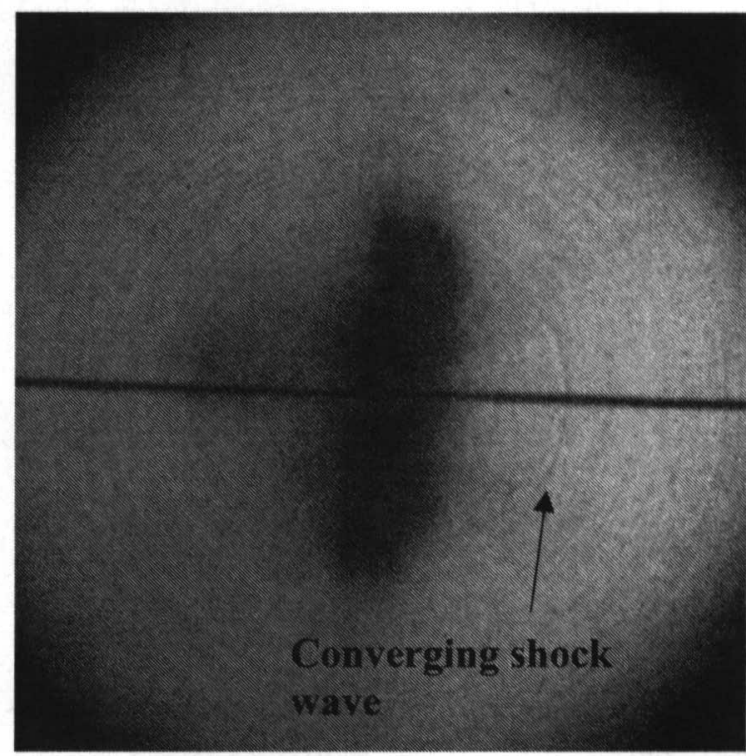

Fig. 4 One frame of high speed shadowgraph of converging spherical shock wave after reflection from the test section, $250 \mu$ s after two sides ignition of 10.0 $\mathrm{mg}$ silver azide charge at ambient pressure $100.65 \mathrm{kPa}$ 